\title{
Estimated transmissibility and impact of SARS-CoV-2 lineage B.1.1.7 in England
}

\author{
Nicholas G. Davies ${ }^{1 *}$, Sam Abbott ${ }^{1} \uparrow$, Rosanna C. Barnard ${ }^{1} \uparrow$, Christopher I. Jarvis ${ }^{1} \uparrow$, \\ Adam J. Kucharski ${ }^{1} \uparrow$, James D. Munday ${ }^{1} \uparrow$, Carl A. B. Pearson ${ }^{1} \uparrow$, Timothy W. Russell ${ }^{1} \uparrow$, \\ Damien C. Tully ${ }^{1} \uparrow$, Alex D. Washburne ${ }^{2} \uparrow$, Tom Wenseleers ${ }^{3} \uparrow$, Amy Gimma $^{1}$, William Waites ${ }^{1}$, \\ Kerry L. M. Wong ${ }^{1}$, Kevin van Zandvoort ${ }^{1}$, Justin D. Silverman ${ }^{4}$, CMMID COVID-19 Working \\ Group ${ }^{1}$, COVID-19 Genomics UK (COG-UK) Consortium $\$$, Karla Diaz-Ordaz ${ }^{5}$, Ruth Keogh $^{5}$, \\ Rosalind M. Eggo' ${ }^{1}$, Sebastian Funk ${ }^{1}$, Mark Jit' ${ }^{1}$, Katherine E. Atkins ${ }^{1,6}$, W. John Edmunds ${ }^{1}$
}

\begin{abstract}
${ }^{1}$ Centre for Mathematical Modelling of Infectious Diseases, London School of Hygiene and Tropical Medicine, London, UK. ${ }^{2}$ Selva Analytics LLC, Bozeman, MT, USA. ${ }^{3}$ Lab of Socioecology and Social Evolution, KU Leuven, Leuven, Belgium. ${ }^{4}$ College of Information Science and Technology, Pennsylvania State University, University Park, PA, USA. ${ }^{5}$ Centre for Statistical Methodology and Department of Medical Statistics, London School of Hygiene and Tropical Medicine, London, UK. ${ }^{6} \mathrm{Centre}$ for Global Health, Usher Institute of Population Health Sciences and Informatics, University of Edinburgh, Edinburgh, UK.

${ }^{*}$ Corresponding author. Email: nicholas.davies@Ishtm.ac.uk

$\dagger$ These authors contributed equally to this work.

$\ddagger$ See supplementary materials for list of consortium members and affiliations.

A novel SARS-CoV-2 variant, VOC 202012/01 (lineage B.1.1.7), emerged in southeast England in November 2020 and is rapidly spreading toward fixation. Using a variety of statistical and dynamic modelling approaches, we estimate that this variant has a $43-90 \%$ (range of 95\% credible intervals $38-130 \%$ ) higher reproduction number than preexisting variants. A fitted two-strain dynamic transmission model shows that VOC 202012/01 will lead to large resurgences of COVID-19 cases. Without stringent control measures, including limited closure of educational institutions and a greatly accelerated vaccine roll-out, COVID-19 hospitalisations and deaths across England in 2021 will exceed those in 2020. Concerningly, VOC 202012/01 has spread globally and exhibits a similar transmission increase (59-74\%) in Denmark, Switzerland, and the United States.
\end{abstract}

In December 2020, evidence began to emerge that a novel SARS-CoV-2 variant, Variant of Concern 202012/01 (lineage B.1.1.7, henceforth VOC 202012/01), was rapidly outcompeting preexisting variants in southeast England (1). The variant increased in incidence during a national lockdown in November 2020, which was mandated in response to a previous and unrelated surge in COVID-19 cases, and continued to spread following the lockdown despite ongoing restrictions in many of the most affected areas. Concern over this variant led the UK government to enact stronger restrictions in these regions on 20 December 2020, and eventually to impose a third national lockdown on 5 January 2021. As of 15 February 2021, VOC 202012/01 comprises roughly $95 \%$ of new SARS-CoV-2 infections in England, and has now been identified in at least 82 countries (2). Our current understanding of effective pharmaceutical and nonpharmaceutical control of SARS-CoV-2 does not reflect the epidemiological and clinical characteristics of VOC
202012/01. Estimates of the growth rate, disease severity, and impact of this novel variant are crucial for informing rapid policy responses to this potential threat.

\section{Characteristics of the new variant}

VOC 202012/01 is defined by 17 mutations (14 nonsynonymous point mutations and 3 deletions), of which eight are in the spike protein, which mediates SARS-CoV-2 attachment and entry into human cells. At least three mutations potentially affect viral function. Mutation N501Y is a key contact residue in the receptor binding domain and enhances virus binding affinity to human angiotensin converting enzyme 2 (ACE2) $(3,4)$. Mutation $\mathrm{P} 681 \mathrm{H}$ is immediately adjacent to the furin cleavage site in spike, a known region of importance for infection and transmission $(5,6)$. Deletion $\Delta \mathrm{H} 69 / \Delta \mathrm{V} 70$ in spike has arisen in multiple independent lineages of SARS-CoV-2, is linked to immune escape in immunocompromised patients, and enhances viral infectivity 
in vitro $(7,8)$. This deletion is also responsible for certain commercial testing kits failing to detect the spike glycoprotein gene, with genomic data confirming these $\mathrm{S}$ gene target failures in England are now overwhelmingly due to the new variant (1).

The proportion of COVID-19 cases attributable to VOC 202012/01 is rapidly increasing in all regions of England, following an initial expansion in the South East (Fig. 1A), and is spreading at comparable rates among males and females and across age and socioeconomic strata (Fig. 1B). One potential explanation for the spread of VOC 202012/01 within England is a founder effect: that is, if certain regions had higher levels of transmission as a result of more social interactions, variants that were more prevalent within these regions could become more common overall. Changes in social contact patterns correlate closely with changes in transmission (9) (Fig. 1, C and D) and with COVID-19 burden in England (10). However, we did not find substantial differences in social interactions between regions of high and low VOC 202012/01 prevalence, as measured by Google mobility (11) and social contact survey data (12) from September to December 2020 (Fig. 1, E and F). Therefore, the apparent decoupling between contact rates and transmission in late 2020 may suggest altered transmission characteristics for VOC 202012/01.

\section{Measuring the new variant's growth rate}

VOC 202012/01 appears unmatched in its ability to outcompete other SARS-CoV-2 lineages in England. Analyzing the COG-UK dataset (13), which comprises over 150,000 sequenced SARS-CoV-2 samples from across the UK, we found that the relative population growth rate of VOC 202012/01 in the first 31 days following its initial phylogenetic observation was higher than that of all 307 other lineages with enough observations to obtain reliable growth-rate estimates (Fig. 2A and fig. S1). While the relative growth rate of VOC 202012/01 has declined slightly over time, it remains among the highest of any lineage as a function of lineage age (Fig. 2B), and the lineage continues to expand.

To quantify the growth advantage of VOC 202012/01, we performed a series of multinomial and logistic regression analyses on COG-UK data. A time-varying multinomial spline model estimates an increased growth rate for VOC $202012 / 01$ of +0.104 days $^{-1}$ (95\% CI $\left.0.100-0.108\right)$ relative to the previously dominant lineage, B.1.177 (Table 1, model 1a; Fig. 2C and figs. S2 and S3). Assuming a generation interval of 5.5 days (14), this corresponds to a $77 \%$ (73-81\%) increase in the reproduction number $R$. The growth advantage of VOC 202012/01 persists under more conservative model assumptions (Table 1, model 1b; fig. S4), is consistent across all regions of the UK (table S1, model 2a; fig. S5), and is similar when measured from $\mathrm{S}$ gene target failures among community COVID-19 tests instead of COG-UK sequence data (Table 1, model 2h; fig. S6). Data from other countries yield similar results: we estimate that $R$ for VOC 202012/01 relative to other lineages is $55 \%(45-66 \%)$ higher in Denmark, 74\% (66-82\%) higher in Switzerland, and 59\% (56$63 \%$ ) higher in the United States, with consistent rates of displacement across regions within each country (Table 1, models 3a-c; figs. S6 and S7).

As an alternative approach, we performed a regression analysis of previously-estimated reproduction numbers from case data against the frequency of $\mathrm{S}$ gene target failure in English upper-tier local authorities (Fig. 2D), using local control policies and mobility data as covariates and including a time-varying spline to capture any unmeasured confounders. This yielded an estimated increase in $R$ for VOC $202012 / 01$ of $43 \%(38-48 \%)$, increasing to a $57 \%(52-62 \%)$ increase if the spline was not included (Table 1, model 4ab). The various statistical models we fitted yield slightly different estimates for the growth rate of VOC 202012/01, reflecting different assumptions and model structures, but all identify a substantially increased growth rate (table S1).

\section{Mechanistic hypotheses for the rapid spread}

To understand possible biological mechanisms for why VOC 202012/01 spreads more quickly than preexisting variants, we extended an age- and regionally-structured mathematical model of SARS-CoV-2 transmission $(10,15)$ to consider two co-circulating variants (fig. S8 and tables S2 and S3). The model uses Google mobility data (11), validated by social contact surveys (10), to capture changes in contact patterns over time for each region of England. We created five versions of the model, each including one alternative parameter capturing a potential mechanism.

The hypotheses we tested are as follows. First, observations of lower $\mathrm{Ct}$ values (16-18)-i.e., higher viral loadsupport that VOC may be more transmissible per contact with an infectious person than preexisting variants (hypothesis 1). Second, longitudinal testing data (17) suggest that VOC may be associated with a longer period of viral shedding, and hence a potentially longer infectious period (hypothesis 2). Third, the $\Delta \mathrm{H} 69 / \Delta \mathrm{V} 70$ deletion in spike contributed to immune escape in an immunocompromised patient (7), potentially suggesting that immunity to preexisting variants affords reduced protection against infection with VOC (hypothesis 3). Fourth, that VOC initially spread during the November 2020 lockdown in England, during which schools were open, suggests that children may be more susceptible to infection with VOC than with preexisting variants (hypothesis 4). Children are typically less susceptible to SARS-CoV-2 infection than adults $(19,20)$, possibly because of immune cross-protection due to other human coronaviruses (21), which could be less protective 
against VOC. Finally, VOC could have a shorter generation time than preexisting variants (hypothesis 5). A shorter generation time could account for an increased growth rate without requiring a higher reproduction number, which would make control of VOC 202012/01 via social distancing measures relatively easier to achieve.

We fit each model to time series of COVID-19 deaths, hospital admissions, hospital and ICU bed occupancy, PCR prevalence, seroprevalence, and the proportion of community SARS-CoV-2 tests with $\mathrm{S}$ gene target failure across the three most heavily affected NHS England regions, over the period of 1 March-24 December 2020 (Fig. 3 and figs. S9 to S14). We assess models using Deviance Information Criteria (DIC) and by comparing model predictions to observed data for the 14 days following the fitting period (i.e., 25 December 2020-7 January 2021). Of the five hypotheses assessed, hypothesis 1 (increased transmissibility) had the lowest (i.e., best) combined DIC and predictive deviance. Hypotheses 2 (longer infectious period) and 4 (increased susceptibility in children) also fitted the data well, although hypothesis 4 is not well supported by household secondary attack rate data (fig. S15) or by age-specific patterns of S gene target failure in the community (fig. S16), neither of which identify a substantial increase in susceptibility among children. Hypotheses 3 (immune escape) and 5 (shorter generation time) fit poorly (Fig. 3A and table S4). In particular, hypothesis 5 predicted that the relative frequency of VOC 202012/01 should have dropped during stringent restrictions in late December 2020, because when two variants have the same $R_{t}<1$ but different generation times, infections decline faster for the variant with the shorter generation time.

We fitted a combined model incorporating the five hypotheses above, but it was not able to identify a single consistent mechanism across NHS England regions, demonstrating that a wide range of parameter values are compatible with the observed growth rate of VOC 202012/01 (fig. S14). Based on our analysis, we identify increased transmissibility as the most parsimonious model, but emphasize that the five mechanisms explored here are not mutually exclusive and may be operating in concert.

The increased transmissibility model does not identify a clear increase or decrease in the severity of disease associated with VOC 202012/01, finding similar odds of hospitalisation (estimated odds ratio of hospitalisation given infection, 0.92 [95\% credible intervals 0.77-1.10]), critical illness (OR 0.90 [0.58-1.40]), and death (OR 0.90 [0.68-1.20]), based upon fitting to the three most heavily affected NHS England regions (Fig. 3B). These estimates should be treated with caution, as we would not expect to identify a clear signal of severity when fitting to data up to 24 December 2020, given delays between infection and hospitalization or death. However, the fitted model finds strong evidence of higher rela- tive transmissibility, estimated at $65 \%$ (95\% CrI: $39-93 \%$ ) higher than preexisting variants for the three most heavily affected NHS England regions, or 82\% (43-130\%) when estimated across all seven NHS England regions (Table 1, model 5a). These estimates of increased transmissibility are consistent with our statistical estimates and with a previous estimate of a $70 \%$ increased reproduction number for VOC 202012/01 (16). This model reproduces observed epidemiological dynamics for VOC 202012/01 (Fig. 3C and fig. S17). Without the introduction of a new variant with a higher growth rate, the model is unable to reproduce observed dynamics (Fig. 3, D and E, and figs. S17 to S19), further highlighting that changing contact patterns do not explain the spread of VOC 202012/01.

\section{Implications for COVID-19 dynamics in England}

Using the best-performing transmission model (increased transmissibility) fitted to all seven NHS England regions, we compared projected epidemic dynamics under different assumptions about control measures from mid-December 2020 to the end of June 2021. We compared four scenarios for non-pharmaceutical interventions (NPIs) introduced on 1 January 2021: (i) a moderate-stringency scenario with mobility levels as observed in the first half of October 2020; (ii) a high-stringency scenario with mobility levels as observed during the second national lockdown in England in November 2020, with schools open; (iii) the same high-stringency scenario, but with schools closed until 15 February 2021; and (iv) a very high-stringency scenario with mobility levels as observed during the first national lockdown in early April 2020, with schools closed (fig. S20). In combination with these NPI scenarios, we considered three vaccination scenarios: no vaccinations; 200,000 vaccinations per week; and 2 million vaccinations per week. We assumed that vaccine rollout starts on 1 January 2021 and that vaccinated individuals have a $95 \%$ lower probability of disease and a $60 \%$ lower probability of infection than unvaccinated individuals. For simplicity, we assumed that vaccine protection was conferred immediately upon receipt of one vaccine dose. Note that these projections serve as indicative scenarios rather than formal predictive forecasts.

Regardless of control measures, all regions of England were projected to experience a new wave of COVID-19 cases and deaths in early 2021, peaking in February 2021 if no substantial control measures are introduced, or in midJanuary 2021 if strong control measures succeeded in reducing $R$ below 1 (Fig. 4A). In the absence of substantial vaccine roll-out, the number of COVID-19 cases, hospitalisations, ICU admissions and deaths in 2021 were predicted to exceed those in 2020, even with stringent NPIs in place (Table 2). Implementing more stringent measures in January 2021 (scenarios iii and iv) led to a larger rebound in cases when 
simulated restrictions were lifted in March 2021, particularly in those regions that had been least affected up to December 2020 (fig. S21). However, these more stringent measures may buy time to reach more widespread population immunity through vaccination. Vaccine roll-out further mitigates transmission, although the impact of vaccinating 200,000 people per week-similar in magnitude to the rates reached in December 2020-was relatively small (Fig. 4B and fig. S22). An accelerated uptake of 2 million people fully vaccinated per week (i.e., 4 million doses for a two-dose vaccine) had a much more substantial impact (Fig. 4C and fig. S23). However, accelerated vaccine roll-out has a relatively limited impact on peak burden, as the peak is largely mediated by the stringency of NPIs enacted in January 2021, before vaccination has much of an impact. The primary benefit of accelerated vaccine roll-out lies in helping to avert a resurgence of cases following the relaxation of NPIs, and in reducing transmission after the peak burden has already been reached.

As a sensitivity analysis, we also ran model projections with a seasonal component such that transmission is $20 \%$ higher in the winter than in the summer (22), but this did not qualitatively affect our results (fig. S24 and table S5).

\section{Discussion}

Combining multiple behavioral and epidemiological data sources with statistical and dynamic modelling, we estimated that the novel SARS-CoV-2 variant VOC 202012/01 has a $43-90 \%$ (range of $95 \%$ credible intervals $38-130 \%$ ) higher reproduction number than preexisting variants of SARSCoV-2 in England, assuming no changes to the generation interval. Based on early population-level data, we were unable to identify whether the new variant is associated with higher disease severity. Theoretical considerations suggest that mutations conferring increased transmissibility to pathogens may be inextricably linked to reduced severity of disease (23). However, this framework assumes that a long history of adaptive evolution has rendered mutations yielding increased transmissibility inaccessible without a decrease in virulence, which does not obviously hold for a recently emerged human pathogen such as SARS-CoV-2. Regardless, without strengthened controls, there is a clear risk that future epidemic waves may be larger-and hence associated with greater burden-than previous waves. The UK government initiated a third national lockdown on 5 January 2021 in response to the rapid spread of VOC 202012/01, including school closures. Educational settings are among the largest institutions linked to SARS-CoV-2 clusters that remained open during the November 2020 lockdown (24), which means the enacted school and university closures may substantially assist in reducing the burden of COVID-19 in early 2021.
The rise in transmission from VOC 202012/01 has crucial implications for vaccination. First, it means prompt and efficient vaccine delivery and distribution is even more important to reduce the impact of the epidemic in the near future. Increased transmission resulting from VOC 202012/01 will raise the herd immunity threshold, meaning the potential burden of SARS-CoV-2 is larger and higher vaccine coverage will be required to achieve herd immunity. It is therefore extremely concerning that VOC 202012/01 has spread to at least 82 countries globally (2). Although VOC 202012/01 was first identified in England, a rapidly spreading variant has also been detected in South Africa $(25,26)$, where there has been a marked increase in transmission in late 2020. Another variant exhibiting immune escape has emerged in Brazil $(27,28)$. Thus, vaccination timelines will also be a crucial determinant of future burden in other countries where similar new variants are present. Second, there is a need to assess how VOC 202012/01 and other emerging lineages affect the efficacy of vaccines $(29,30)$. Vaccine developers may need to consider developing formulations with variant sequences, and powering post-licensure studies to detect differences in efficacy between the preexisting and new variants. Licensing authorities may need to clarify abbreviated pathways to marketing for vaccines that involve altering strain formulation without any other changes to their composition.

There are limitations to our analysis. We have considered a small number of intervention and vaccination scenarios, which should not be regarded as the only available options for policymakers. Our transmission model does not explicitly capture care home or hospital transmission of SARS-CoV-2, and is fit to each region of England separately rather than pooling information across regions and explicitly modelling transmission between regions. There are also uncertainties in the choice of model used to generate these predictions, and the exact choice will yield differences in the measures needed to control the epidemic. We note that even without increased susceptibility of children to VOC $202012 / 01$, the more efficient spread of the variant implies that the difficult societal decision of closing schools will be a key public health question for multiple countries in the months ahead.

We only assess relative support in the data for the mechanistic hypotheses proposed, but there may be other plausible mechanisms driving the resurgence of cases that we did not consider, and we have not identified the specific combination of mechanisms driving the increased transmission of VOC 202012/01. We identify increased transmissibility as the most parsimonious mechanistic explanation for the higher growth rate of VOC 202012/01, but a longer infectious period also fits the data well (table S4) and is supported by longitudinal testing data (17). Our conclusions about 
school closures were based on the assumption that children had reduced susceptibility and infectiousness compared to adults (19), but the precise values of these parameters and the impact of school closures remains the subject of scientific debate (31). We based our assumptions about the efficacy of NPIs on the measured impact on mobility of previous national lockdowns in England, but the impact of policy options cannot be predicted with certainty.

Despite these limitations, we found strong evidence that VOC $202012 / 01$ is spreading substantially faster than preexisting SARS-CoV-2 variants. Our modelling analysis suggests this difference could be explained by an overall higher infectiousness of VOC 202012/01, but not by a shorter generation time or immune escape alone. Further experimental work will provide insight into the biological mechanisms for our observations, but given our projections of a rapid rise in incidence from VOC 202012/01-and the detection of other novel and highly-transmissible variants (25-28)-there is an urgent need to consider what new approaches may be required to sufficiently reduce the ongoing transmission of SARS-CoV-2.

\section{Materials and methods}

Summary of control measures in England in late 2020

Following a resurgence of cases in September and October 2020, a second national lockdown was implemented in England, from 5 November to 2 December 2020. Restrictions included a stay-at-home order with exemptions for exercise, essential shopping, obtaining or providing medical care, education and work for those unable to work from home. Schools were kept open. Non-essential shops, retail and leisure venues were required to close. Pubs, bars and restaurants were allowed to offer takeaway services only. Following the second national lockdown, regions in England were assigned to tiered local restrictions according to medium, high and very high alert levels (Tiers 1, 2 and 3). In response to rising cases in southeast England and concerns over VOC 202012/01, the UK government announced on 19 December 2020 that a number of regions in southeast England would be placed into a new, more stringent 'Tier 4', corresponding to a Stay at Home alert level. Tier 4 restrictions were broadly similar to the second national lockdown restrictions. As cases continued to rise and VOC 202012/01 spread throughout England, on 5 January 2021 a third national lockdown was introduced in England, with schools and universities closed and individuals advised to stay at home, with measures to be kept in place until at least mid-February 2021.

\section{Data sources}

To assess the spread of VOC 202012/01 in the United Kingdom, we used publicly-available sequencing-based data from the COG-UK Consortium (13) (5 February 2020-6 January 2021) and Pillar 2 SARS-CoV-2 testing data provided by Public Health England (1 October 2020-7 January 2021) for estimating the frequency of $\mathrm{S}$ gene target failure in England. COG-UK sequencing data for Northern Ireland were excluded due to low sample sizes.

To assess the spread of VOC 202012/01 in Denmark, Switzerland and the USA, we used publicly available sequence data giving the incidence of VOC 202012/01 aggregated by week and region provided by the Danish Covid-19 Genome Consortium and the Statens Serum Institut (32) (15 October 2020-28 January 2021), sequence and RT-PCR 501Y.V1 rescreening data giving the incidence of VOC 202012/01 in different regions of Switzerland provided by Christian Althaus and Tanja Stadler and the Geneva University Hospitals, the Swiss Viollier Sequencing Consortium from ETH Zürich, the Risch laboratory, the University Hospital Basel, the Institute for Infectious Diseases, University of Bern and the Swiss National Covid-19 Science Task Force $(33,34)$ (2 November 2020-11 Feb 2021), and publicly available US nation-wide Helix SARS-CoV-2 Surveillance data, comprising both S-gene target failure data and randomly selected S-negative samples that were sequenced to infer the proportion of S-negative samples that were the $\operatorname{VOC}(35,36)$ (6 September-11 February 2020).

To estimate mobility, we used anonymised mobility data collected from smartphone users by Google Community Mobility (11). Percentage change in mobility per day was calculated for each lower-tier local authority in England and a generalised additive model with a spline for time was fitted to these observations to provide a smoothed effect of the change in mobility over time (Fig. 1C).

To estimate social contact rates (Fig. 1D), we used data on reported social contacts from the CoMix survey (12), which is a weekly survey of face-to-face contact patterns, taken from a sample of approximately 2500 individuals broadly representative of the UK population with respect to age and geographical location. We calculated the distribution of contacts using 1000 bootstrap samples with replacement from the raw data. Bootstrap samples were calculated at the participant level, then all observations for those participants are included in a sample to respect the correlation structure of the data. We collect data in two panels which alternate weekly, therefore we calculated the mean smoothed over the 2 week intervals to give a larger number of participants per estimate and account for panel effects. We calculated the mean number of contacts (face to face conversational contact or physical contact) in the settings "home", "work", "education" (including childcare, nurseries, schools and universities and colleges), and "other" settings. We calculate the mean contacts by age group and area of residence (those areas which were subsequently 
placed under Tier 4 restrictions on 20 December 2020 as they were experiencing high and rapidly increasing incidence, and those areas of England that were not placed under these restrictions). The mean number of contacts is influenced by a few individuals who report very high numbers of contacts (often in a work context). The means shown here are calculated based on truncating the maximum number of contacts recorded at 200 per individual per day. We compare $R_{t}$ estimates derived from CoMix (12) to those derived from the REACT-1 prevalence survey (9) for England.

\section{Statistical methods in brief}

Growth of VOC 202012/01 following initial phylogenetic observation. For each lineage $i$ in the COG-UK dataset, we pool the number of sequences observed within that lineage across the UK for every day, $t$, yielding integer-valued sequence counts $N(i, t)$. We estimate the time-varying exponential growth rates of cases of each strain, $r(i, t)$, using a negative binomial state-space model correcting for day-ofweek effects whose dispersion parameter was optimized for each strain by marginal likelihood maximization. We defined the relativized growth rate of a lineage $i$ at time $t$ as $\rho(i, t)=[r(i, t)-\bar{r}(t)] / \sigma_{r}(t)$, where $\bar{r}(t)$ is the average growth rate of all circulating strains at time $t$ and $\sigma_{r}(t)$ the standard deviation of growth rates across all lineages at time $t$, such that $\rho(i, t)$ is analogous to a z-statistic or Waldtype statistic and allows comparison of growth rate differences across time when the average growth rate and scale of growth rate differences varies.

Competitive advantage and increased growth rate of VOC-202012/01. To estimate the increase in growth rate of VOC 202012/01, we fitted a set of multinomial and binomial generalized linear mixed models (GLMMs), in which we estimated the rate by which the VOC displaces other resident SARS-CoV-2 variants across different regions in the UK, based on both the COG-UK sequence data as well as the $\mathrm{S}$ gene target failure data. In the analysis of the $S$ gene target failure data, binomial counts were adjusted for the true positive rate. For comparison, we also calculated the growth advantage of the VOC in Denmark, Switzerland and the US based on both sequencing and S gene target failure data. All models took into account sample date and region plus, if desired, their interaction, and all mixed models took into account possible overdispersion and for the UK further included local-tier local authority as a random intercept. From these models, we estimated the difference in Malthusian growth rate between other competing variants $\Delta r$, as well as the expected multiplicative increase in basic reproduction number $R_{t}$ and infectiousness, assuming unaltered generation time, which can be shown to be equal to $\exp (\Delta r . T)$, where $T$ is the mean generation interval. The multiplicative increase being equal to $\exp (\Delta r . T)$ is an approximation that holds for a delta-distributed generation interval, but we show in the Supplementary Material that this is a good approximation for the gamma-distributed generation interval that we assume. In our calculations, we used estimated SARS-CoV-2 mean generation times $T$ of either 5.5 days (14) (Table 1 ) or 3.6 days $(37,38)$ (table S1).

$R_{t}$ analysis. We calculated the weekly proportion of positive tests that were S-gene negative out of all positive tests that tested for the S-gene by English upper-tier local authority. We used reproduction number estimates obtained using the method described in (37) and (39) and implemented in the EpiNow2 $\mathrm{R}$ package (40), downloaded from https://github.com/epiforecasts/covid-rt-estimates/blob/ master/subnational/united-kingdom-

local/cases/summary/rt.csv. We then built a separate model of the expected reproduction number in UTLA $i$ during week $t$ starting in the week beginning the 5 October 2020 as a function of local restrictions, mobility indicators, residual temporal variation, and proportion of positive tests with $\mathrm{S}$ gene target failure. The residual temporal variation is modeled either as a region-specific thin-plate regression spline ("Regional time-varying") or a static regional parameter ("Regional static"). The key estimand is the relative change in reproduction number in the presence of $\mathrm{S}$ gene target failure that is not explained by any of the other variables.

\section{Transmission dynamic model}

We extended a previously developed modelling framework structured by age (in 5-year age bands, with no births, deaths, or aging due to the short timescales modeled) and by geographical region $(10,15)$ to include two variants of SARS-CoV-2 (VOC 202012/01 and non-VOC 202012/01). The model is a discrete-time deterministic compartmental model which allows for arbitrary delay distributions for transitions between compartments. We fitted this model to multiple regionally-stratified data sources across the 7 NHS England regions as previously: deaths, hospital admissions, hospital bed occupancy, ICU bed occupancy, daily incidence of new infections, PCR prevalence of active infection, seroprevalence, and the daily frequency of VOC 202012/01 across each of the regions as measured by $\mathrm{S}$ gene target failure frequency corrected for false positives. The model assumes that individuals with clinical symptoms are more infectious than individuals with subclinical infection (19). We assume that vaccinated individuals have a lower probability of both clinical and subclinical infection (fig. S9), but that vaccinated individuals who do develop clinical or subclinical infection are as infectious as unvaccinated individuals with clinical or subclinical infection. To model school closure, we removed all school contacts from our contact matrix based upon 
POLYMOD data and varying over time according to Google Mobility indices, as described previously (10). See supplementary materials for details of Bayesian inference including likelihood functions and prior distributions.

Our individual transmission model fits to separate NHS regions of England produce independent estimates of parameters such as relative transmissibility and differences in odds of hospitalisation or death resulting from infection with VOC 202012/01. In order to produce overall estimates for these parameters, we model posterior distributions from individual NHS regions as draws from a mixture distribution, comprising a normally-distributed top-level distribution from which central estimates for each NHS region are drawn. We report the mean and credible intervals of the top-level distribution when reporting model posterior estimates for England.

In model fitting, we assume that our deterministic transmission model approximates the expectation over stochastic epidemic dynamics. This is not exact (41) but the error in this approximation is small for the population-level processes we are modelling, as it decays with $1 / N(42)$. This approach is well developed for state space models of communicable disease dynamics that fit an epidemic process to observed data via a stochastic observation process.

\section{Apparent growth of VOC 202012/01 not a result of testing artefacts}

The apparent frequency of VOC 202012/01 could be inflated relative to reality if this variant leads to increased testseeking behavior (e.g., if it leads to a higher rate of symptoms than preexisting variants). However, this would not explain the growth in the relative frequency of VOC 202012/01 over time. Mathematically, if variant 1 has growth rate $r_{1}$ and variant 2 has growth rate $r_{2}$, the relative frequency over time is $a_{2} \exp \left(r_{2} t\right) /\left(a_{1} \exp \left(r_{1} t\right)+a_{2} \exp \left(r_{2} t\right)\right)$, where $a_{1}$ and $a_{2}$ are the frequency of variant 1 and 2, respectively, at time $t=0$. However, if variant 1 has probability $x$ of being reported and variant 2 has probability $y$, and both have growth rate $r$, the relative frequency over time is $a_{2} y \exp (r t)$ / $\left(a_{1} x \exp (r t)+a_{2} y \exp (r t)\right)$, which is constant.

\section{REFERENCES AND NOTES}

1. Public Health England, "Investigation of novel SARS-COV-2 variant: Variant of Concern 202012/01" (2020);

www.gov.uk/government/publications/investigation-of-novel-sars-cov-2variant-variant-of-concern-20201201.

2. Á. O'Toole, "Tracking the international spread of SARS-CoV-2 lineages B.1.1.7 and B.1.351/501Y-V2" (2021); https://virological.org/t/tracking-the-internationalspread-of-sars-cov-2-lineages-b-1-1-7-and-b-1-351-501y-v2/592.

3. H. Gu, Q. Chen, G. Yang, L. He, H. Fan, Y.-Q. Deng, Y. Wang, Y. Teng, Z. Zhao, Y. Cui, Y. Li, X.-F. Li, J. Li, N.-N. Zhang, X. Yang, S. Chen, Y. Guo, G. Zhao, X. Wang, D.-Y. Luo, H. Wang, X. Yang, Y. Li, G. Han, Y. He, X. Zhou, S. Geng, X. Sheng, S. Jiang, S. Sun, C.-F. Qin, Y. Zhou, Adaptation of SARS-CoV-2 in BALB/c mice for testing vaccine efficacy. Science 369, 1603-1607 (2020). Medline

4. T. N. Starr, A. J. Greaney, S. K. Hilton, D. Ellis, K. H. D. Crawford, A. S. Dingens, M. J. Navarro, J. E. Bowen, M. A. Tortorici, A. C. Walls, N. P. King, D. Veesler, J. D. Bloom, Deep Mutational Scanning of SARS-CoV-2 Receptor Binding Domain Reveals Constraints on Folding and ACE2 Binding. Cell 182, 1295-1310.e20 (2020). doi:10.1016/i.cell.2020.08.012 Medline

5. M. Hoffmann, H. Kleine-Weber, S. Pöhlmann, A Multibasic Cleavage Site in the Spike Protein of SARS-CoV-2 Is Essential for Infection of Human Lung Cells. Mol. Cell 78, 779-784.e5 (2020). doi:10.1016/j.molcel.2020.04.022 Medline

6. T. P. Peacock et al., The furin cleavage site of SARS-CoV-2 spike protein is a key determinant for transmission due to enhanced replication in airway cells. bioRxiv 10.1101/2020.09.30.318311 [preprint]. 30 September 2020.

7. S. A. Kemp et al., Neutralising antibodies drive Spike mediated SARS-CoV-2 evasion. medRxiv 10.1101/2020.12.05.20241927v2 [preprint]. 19 December 2020

8. S. Kemp et al., Recurrent emergence and transmission of a SARS-CoV-2 Spike deletion $\triangle H$ 69/V70. bioRxiv 10.1101/2020.12.14.422555 [preprint]. 21 December 2020.

9. "Real-Time Assessment of Community Transmission findings" (2021); www.imperial.ac.uk/medicine/research-and-impact/groups/react-study/realtime-assessment-of-community-transmission-findings/.

10. N. G. Davies, R. C. Barnard, C. I. Jarvis, T. W. Russell, M. G. Semple, M. Jit, W. J. Edmunds, Association of tiered restrictions and a second lockdown with COVID19 deaths and hospital admissions in England: A modelling study. Lancet Infect. Dis. S1473-3099(20)30984-1 (2020). doi:10.1016/S1473-3099(20)30984-1 Medline

11. Google, COVID-19 Community Mobility Reports (2021); www.google.com/covid19/mobility/.

12. C. I. Jarvis, K. Van Zandvoort, A. Gimma, K. Prem, P. Klepac, G. J. Rubin, W. J. Edmunds, CMMID COVID-19 Working Group, Quantifying the impact of physical distance measures on the transmission of COVID-19 in the UK. BMC Med. 18, 124 (2020). doi:10.1186/s12916-020-01597-8 Medline

13. COG-UK Consortium, Public Data \& Analysis (2021); https://www.cogconsortium.uk/tools-analysis/public-data-analysis-2/.

14. L. Ferretti et al., The timing of COVID-19 transmission. medRxiv 10.1101/2020.09.04.20188516 [preprint]. 7 September 2020.

15. N. G. Davies, A. J. Kucharski, R. M. Eggo, A. Gimma, W. J. Edmunds, T. Jombart, K. O'Reilly, A. Endo, J. Hellewell, E. S. Nightingale, B. J. Quilty, C. I. Jarvis, T. W. Russell, P. Klepac, N. I. Bosse, S. Funk, S. Abbott, G. F. Medley, H. Gibbs, C. A. B. Pearson, S. Flasche, M. Jit, S. Clifford, K. Prem, C. Diamond, J. Emery, A. K. Deol, S. R. Procter, K. van Zandvoort, Y. F. Sun, J. D. Munday, A. Rosello, M. Auzenbergs, G. Knight, R. M. G. J. Houben, Y. Liu, Effects of non-pharmaceutical interventions on COVID-19 cases, deaths, and demand for hospital services in the UK: A modelling study. Lancet Public Health 5, e375-e385 (2020). doi:10.1016/S2468-2667(20)30133-X Medline

16. New and Emerging Respiratory Virus Threats Advisory Group, "NERVTAG meeting on SARS-CoV-2 variant under investigation VUI-202012/01" (2020); https://khub.net/documents/135939561/338928724/SARS-CoV$2+$ variant+under+investigation\%2C+meeting+minutes.pdf/962e866b-161f2fd5-1030-32b6ab467896.

17. S. Kissler, J. R. Fauver, C. Mack, C. G. Tai, M. I. Breban, A. E. Watkins, R. M. Samant, D. J. Anderson, D. D. Ho, N. D. Grubaugh, Y. Grad, "Densely sampled viral trajectories suggest longer duration of acute infection with B.1.1.7 variant relative to non-B.1.1.7 SARS-CoV-2" (preprint, 2021); https://dash.harvard.edu/handle/1/37366884.

18. N. G. Davies et al., Increased hazard of death in community-tested cases of SARS-CoV-2 Variant of Concern 202012/01. medRxiv 10.1101/2021.02.01.21250959 [preprint]. 11 February 2021.

19. N. G. Davies, P. Klepac, Y. Liu, K. Prem, M. Jit, R. M. Eggo, CMMID COVID-19 Working Group, Age-dependent effects in the transmission and control of 
COVID-19 epidemics. Nat. Med. 26, 1205-1211 (2020). doi:10.1038/s41591-0200962-9 Medline

20. R. M. Viner, O. T. Mytton, C. Bonell, G. J. Melendez-Torres, J. Ward, L. Hudson, C. Waddington, J. Thomas, S. Russell, F. van der Klis, A. Koirala, S. Ladhani, J. Panovska-Griffiths, N. G. Davies, R. Booy, R. M. Eggo, Susceptibility to SARSCoV-2 Infection Among Children and Adolescents Compared With Adults: A Systematic Review and Meta-analysis. JAMA Pediatr. 175, 143-156 (2021). doi:10.1001/jamapediatrics.2020.4573 Medline

21. K. W. Ng, N. Faulkner, G. H. Cornish, A. Rosa, R. Harvey, S. Hussain, R. Ulferts, C. Earl, A. G. Wrobel, D. J. Benton, C. Roustan, W. Bolland, R. Thompson, A. AguaDoce, P. Hobson, J. Heaney, H. Rickman, S. Paraskevopoulou, C. F. Houlihan, K. Thomson, E. Sanchez, G. Y. Shin, M. J. Spyer, D. Joshi, N. O'Reilly, P. A. Walker, S. Kjaer, A. Riddell, C. Moore, B. R. Jebson, M. Wilkinson, L. R. Marshall, E. C. Rosser, A. Radziszewska, H. Peckham, C. Ciurtin, L. R. Wedderburn, R. Beale, C. Swanton, S. Gandhi, B. Stockinger, J. McCauley, S. J. Gamblin, L. E. McCoy, P. Cherepanov, E. Nastouli, G. Kassiotis, Preexisting and de novo humoral immunity to SARS-CoV-2 in humans. Science 370, 1339-1343 (2020). doi:10.1126/science abel107 Medline

22. S. M. Kissler, C. Tedijanto, E. Goldstein, Y. H. Grad, M. Lipsitch, Projecting the transmission dynamics of SARS-CoV-2 through the postpandemic period. Science 368, 860-868 (2020). doi:10.1126/science.abb5793 Medline

23. S. A. Frank, Models of parasite virulence. Q. Rev. Biol. 71, 37-78 (1996). doi:10.1086/419267 Medline

24. Public Health England, "National flu and COVID-19 surveillance reports" (2020); www.gov.uk/government/statistics/national-flu-and-covid-19-surveillancereports.

25. H. Tegally et al., Emergence and rapid spread of a new severe acute respiratory syndrome-related coronavirus 2 (SARS-CoV-2) lineage with multiple spike mutations in South Africa. medRxiv 10.1101/2020.12.21.20248640 [preprint]. 22 December 2020

26. C. A. B. Pearson, T. W. Russell, N. G. Davies, A. J. Kucharski, CMMID COVID-19 Working Group, W. J. Edmunds, R. M. Eggo, "Estimates of severity and transmissibility of novel SARS-CoV-2 variant 501Y.V2 in South Africa" (CMMID Repository, 2021); https://cmmid.github.io/topics/covid19/sa-novelvariant.html.

27. F. Naveca, C. da Costa, V. Nascimento, V. Souza, A. Corado, F. Nascimento, Á. Costa, D. Duarte, G. Silva, M. Mejía, K. Pessoa, L. Gonçalves, M. J. Brandão, M. Jesus, R. Pinto, M. Silva, T. Mattos, L. Abdalla, J. H. Santos, R. Costa-Filho, G. L. Wallau, M. M. Siqueira, E. Delatorre, T. Gräf, G. Bello, P. C. Resende, "SARS-CoV2 reinfection by the new Variant of Concern (VOC) P.1 in Amazonas, Brazil" (virological.org, 2021); https://virological.org/t/sars-cov-2-reinfection-by-thenew-variant-of-concern-voc-p-1-in-amazonas-brazil/596.

28. E. C. Thomson, L. E. Rosen, J. G. Shepherd, R. Spreafico, A. da Silva Filipe, J. A. Wojcechowskyj, C. Davis, L. Piccoli, D. J. Pascall, J. Dillen, S. Lytras, N. Czudnochowski, R. Shah, M. Meury, N. Jesudason, A. De Marco, K. Li, J. Bassi, A. O'Toole, D. Pinto, R. M. Colquhoun, K. Culap, B. Jackson, F. Zatta, A. Rambaut, S. Jaconi, V. B. Sreenu, J. Nix, I. Zhang, R. F. Jarrett, W. G. Glass, M. Beltramello, K. Nomikou, M. Pizzuto, L. Tong, E. Cameroni, T. I. Croll, N. Johnson, J. Di Iulio, A. Wickenhagen, A. Ceschi, A. M. Harbison, D. Mair, P. Ferrari, K. Smollett, F. Sallusto, S. Carmichael, C. Garzoni, J. Nichols, M. Galli, J. Hughes, A. Riva, A. Ho, M. Schiuma, M. G. Semple, P. J. M. Openshaw, E. Fadda, J. K. Baillie, J. D. Chodera, S. J. Rihn, S. J. Lycett, H. W. Virgin, A. Telenti, D. Corti, D. L. Robertson, G. Snell, ISARIC4C Investigators, COVID-19 Genomics UK (COG-UK) Consortium, Circulating SARS-CoV-2 spike N439K variants maintain fitness while evading antibody-mediated immunity. Cell 10.1016/j.cell.2021.01.037 (2021). doi:10.1016/i.cell.2021.01.037 Medline

29. E. Mahase, Covid-19: Novavax vaccine efficacy is $86 \%$ against UK variant and $60 \%$ against South African variant. BMJ 372, n296 (2021). Medline

30. Z. Wang, F. Schmidt, Y. Weisblum, F. Muecksch, C. O. Barnes, S. Finkin, D. Schaefer-Babajew, M. Cipolla, C. Gaebler, J. A. Lieberman, T. Y. Oliveira, Z. Yang, M. E. Abernathy, K. E. Huey-Tubman, A. Hurley, M. Turroja, K. A. West, K. Gordon, K. G. Millard, V. Ramos, J. D. Silva, J. Xu, R. A. Colbert, R. Patel, J. Dizon,
C. Unson-O'Brien, I. Shimeliovich, A. Gazumyan, M. Caskey, P. J. Bjorkman, R. Casellas, T. Hatziioannou, P. D. Bieniasz, M. C. Nussenzweig, mRNA vaccineelicited antibodies to SARS-CoV-2 and circulating variants. Nature (2021). doi:10.1038/s41586-021-03324-6 Medline

31. K. T. D. Eames, N. L. Tilston, W. J. Edmunds, The impact of school holidays on the social mixing patterns of school children. Epidemics 3, 103-108 (2011). doi:10.1016/i.epidem.2011.03.003 Medline

32. Danish Covid-19 Genome Consortium, "Genomic overview of SARS-CoV-2 in Denmark" (2021); www.covid19genomics.dk/statistics.

33. M. Reichmuth, E. Hodcroft, J. Riou, C. L. Althaus, M. Schibler, I. Eckerle, L. Kaiser F. Suter, M. Huber, A. Trkola, B. Hasse, J. Nilsson, R. Buonomano, A. Wepf, U. Karrer, R. Neher, "Transmission of SARS-CoV-2 variants in Switzerland" (2021); https://ispmbern.github.io/covid-19/variants/.

34. "SARS-CoV-2 variants of concern in Switzerland" (2021); https://ibzshiny.ethz.ch/covidDashboard/variant-plot/index.html.

35. N. L. Washington et al., Genomic epidemiology identifies emergence and rapid transmission of SARS-CoV-2 B.1.1.7 in the United States. medRxiv 10.1101/2021.02.06.21251159 [preprint]. 7 February 2021.

36. Helix OpCo LLC, helix-covid19db (2021); https://github.com/myhelix/helixcovid19db.

37. S. Abbott, J. Hellewell, R. N. Thompson, K. Sherratt, H. P. Gibbs, N. I. Bosse, J. D. Munday, S. Meakin, E. L. Doughty, J. Y. Chun, Y.-W. D. Chan, F. Finger, P. Campbell, A. Endo, C. A. B. Pearson, A. Gimma, T. Russell, S. Flasche, A. J. Kucharski, R. M. Eggo, S. Funk, Estimating the time-varying reproduction number of SARS-CoV-2 using national and subnational case counts. Wellcome Open Res. 5, 112 (2020). doi:10.12688/wellcomeopenres.16006.2

38. T. Ganyani, C. Kremer, D. Chen, A. Torneri, C. Faes, J. Wallinga, N. Hens, Estimating the generation interval for coronavirus disease (COVID-19) based on symptom onset data, March 2020. Eurosurveillance 25, 2000257 (2020). doi:10.2807/1560-7917.ES.2020.25.17.2000257 Medline

39. K. Sherratt et al., Evaluating the use of the reproduction number as an epidemiological tool, using spatio-temporal trends of the Covid-19 outbreak in England. medRxiv 10.1101/2020.10.18.20214585 [preprint]. 20 October 2020.

40. S. Abbott, EpiNow (2020); https://zenodo.org/record/3957490\#.X-JXen7SHE.

41. D. Mollison, Spatial Contact Models for Ecological and Epidemic Spread. J. R. Stat. Soc. Ser. A 39, 283-313 (1977).

42. S. N. Ethier, T. G. Kurtz, Markov Processes: Characterization and Convergence (Wiley, 1986)

43. N. G. Davies, Analysis data and code for "Estimated transmissibility and impact of SARS-CoV-2 lineage B.1.1.7 in England" (Zenodo, 2021); https://zenodo.org/record/4562961.

44. A. Rambaut, E. C. Holmes, Á. O'Toole, V. Hill, J. T. McCrone, C. Ruis, L. du Plessis, 0 . G. Pybus, A dynamic nomenclature proposal for SARS-CoV-2 lineages to assist genomic epidemiology. Nat. Microbiol. 5, 1403-1407 (2020). doi:10.1038/s41564-020-0770-5 Medline

45. J. Helske, KFAS: Exponential family state space models in R. J. Stat. Softw. 78, 10.18637/jss.v078.i10 (2017). doi:10.18637/jss.v078.110

46. J. T. Morton, C. Marotz, A. Washburne, J. Silverman, L. S. Zaramela, A. Edlund, K. Zengler, R. Knight, Establishing microbial composition measurement standards with reference frames. Nat. Commun. 10, 2719 (2019). doi:10.1038/s41467-01910656-5 Medline

47. A. D. Washburne, D. E. Crowley, D. J. Becker, K. R. Manlove, M. L. Childs, R. K Plowright, Percolation models of pathogen spillover. Philos. Trans. R. Soc. London Ser. B 374, 20180331 (2019). doi:10.1098/rstb.2018.0331 Medline

48. M. Travisano, R. E. Lenski, Long-term experimental evolution in Escherichia coli. IV. Targets of selection and the specificity of adaptation. Genetics 143, 15-26 (1996). doi:10.1093/genetics/143.1.15 Medline

49. L.-M. Chevin, On measuring selection in experimental evolution. Biol. Lett. 7 , 


\section{0-213 (2011). doi:10.1098/rsbl.2010.0580 Medline}

50. T. Day, S. Gandon, Applying population-genetic models in theoretical evolutionary epidemiology. Ecol. Lett. 10, 876-888 (2007). doi:10.1111/i.14610248.2007.01091.x Medline

51. S. W. Park, B. M. Bolker, D. Champredon, D. J. D. Earn, M. Li, J. S. Weitz, B. T. Grenfell, J. Dushoff, Reconciling early-outbreak estimates of the basic reproductive number and its uncertainty: Framework and applications to the novel coronavirus (SARS-CoV-2) outbreak. J. R. Soc. Interface 17, 20200144 (2020). doi:10.1098/rsif.2020.0144 Medline

52. J. Wallinga, M. Lipsitch, How generation intervals shape the relationship between growth rates and reproductive numbers. Proc. R. Soc. B 274, 599-604 (2007). doi:10.1098/rspb.2006.3754 Medline

53. E. B. Hodcroft et al., Emergence and spread of a SARS-CoV-2 variant through Europe in the summer of 2020. medRxiv 10.1101/2020.10.25.20219063 [preprint]. 27 November 2020.

54. W. J. Browne, S. V. Subramanian, K. Jones, H. Goldstein, Variance partitioning in multilevel logistic models that exhibit overdispersion. J. R. Stat. Soc. Ser. A 168, 599-613 (2005). doi:10.1111/j.1467-985X.2004.00365.x

55. Comprehensive R Archive Network, "Transformations and link functions in emmeans"; https://cran.r-

project.org/web/packages/emmeans/vignettes/transformations.html\#biasadj.

56. B. Ripley, "Feed-Forward Neural Networks and Multinomial Log-Linear Models" [R package nnet version 7.3-14] (2020); https://cran.rproject.org/package=nnet.

57. Comprehensive R Archive Network, "Models supported by emmeans"; https://cran.r-project.org/web/packages/emmeans/vignettes/models.html.

58. Public Health England; Coronavirus in the UK; https://coronavirus.data.gov.uk/details/healthcare.

59. Office for National Statistics Geography Teams, "Covid 19 local restriction tiers by area"; https://github.com/ONSgeo/Covid19_Tiers.

60. H. Gibbs, C. I. Jarvis, R. M. Eggo, uk_tier_data (2021); DOI: 10.5281/zenodo.4421649.

61. S. Abbott, J. Hickson, H. S. Badr, S. Funk, P. Ellis, P. Monticone, J. D. Munday, J. Allen, C. A. B. Pearson, S. Meakin, EpiNow2; DOI: 10.5281/zenodo.3957489.

62. P.-C. Bürkner, Advanced Bayesian Multilevel Modeling with the R Package brms. R J. 10, 395-411 (2018). doi:10.32614/RJ-2018-017

63. Public Health England, "Investigation of novel SARS-CoV-2 variant Variant of Concern 202012/01: Technical briefing 3" (2021);

https://assets.publishing.service.gov.uk/government/uploads/system/upload s/attachment_data/file/950823/Variant_of_Concern_VOC_202012_01_Techni cal_Briefing_3_-_England.pdf.

64. R. V. Lenth, Estimated Marginal Means, aka Least-Squares Means [R package emmeans version 1.5.3] (2020); https://cran.r-project.org/package=emmeans.

65. C. J. F. T. Braak, C. J. F. Ter Braak, A Markov Chain Monte Carlo version of the genetic algorithm Differential Evolution: Easy Bayesian computing for real parameter spaces. Stat. Comput. 16, 239-249 (2006). doi:10.1007/s11222-006$\underline{8769-1}$

66. A. B. Docherty, E. M. Harrison, C. A. Green, H. E. Hardwick, R. Pius, L. Norman, K. A. Holden, J. M. Read, F. Dondelinger, G. Carson, L. Merson, J. Lee, D. Plotkin, L. Sigfrid, S. Halpin, C. Jackson, C. Gamble, P. W. Horby, J. S. Nguyen-Van-Tam, A. Ho, C. D. Russell, J. Dunning, P. J. Openshaw, J. K. Baillie, M. G. Semple, Features of 20,133 UK patients in hospital with covid-19 using the ISARIC WHO Clinical Characterisation Protocol: Prospective observational cohort study. BMJ 369 , m1985 (2020). doi:10.1136/bmi.m1985 Medline

67. H. Salje, C. Tran Kiem, N. Lefrancq, N. Courtejoie, P. Bosetti, J. Paireau, A. Andronico, N. Hozé, J. Richet, C.-L. Dubost, Y. Le Strat, J. Lessler, D. Levy-Bruhl, A. Fontanet, L. Opatowski, P.-Y. Boelle, S. Cauchemez, Estimating the burden of SARS-CoV-2 in France. Science 369, 208-211 (2020). doi:10.1126/science.abc3517 Medline
68. A. T. Levin, W. P. Hanage, N. Owusu-Boaitey, K. B. Cochran, S. P. Walsh, G. Meyerowitz-Katz, Assessing the age specificity of infection fatality rates for COVID-19: Systematic review, meta-analysis, and public policy implications. Eur. J. Epidemiol. 35, 1123-1138 (2020). doi:10.1007/s10654-020-00698-1 Medline

69. D. Simpson, "Statistical Modeling, Causal Inference, and Social Science" (2018); https://statmodeling.stat.columbia.edu/2018/04/03/justify-my-love/.

70. S. A. Lauer, K. H. Grantz, Q. Bi, F. K. Jones, Q. Zheng, H. R. Meredith, A. S. Azman, N. G. Reich, J. Lessler, The Incubation Period of Coronavirus Disease 2019 (COVID-19) From Publicly Reported Confirmed Cases: Estimation and Application. Ann. Intern. Med. 172, 577-582 (2020). doi:10.7326/M20-0504 Medline

71. Q. Li, X. Guan, P. Wu, X. Wang, L. Zhou, Y. Tong, R. Ren, K. S. M. Leung, E. H. Y. Lau, J. Y. Wong, X. Xing, N. Xiang, Y. Wu, C. Li, Q. Chen, D. Li, T. Liu, J. Zhao, M. Liu, W. Tu, C. Chen, L. Jin, R. Yang, Q. Wang, S. Zhou, R. Wang, H. Liu, Y. Luo, Y. Liu, G. Shao, H. Li, Z. Tao, Y. Yang, Z. Deng, B. Liu, Z. Ma, Y. Zhang, G. Shi, T. T. Y. Lam, J. T. Wu, G. F. Gao, B. J. Cowling, B. Yang, G. M. Leung, Z. Feng, Early Transmission Dynamics in Wuhan, China, of Novel Coronavirus-Infected Pneumonia. N. Engl. J. Med. 382, 1199-1207 (2020). doi:10.1056/NEJMoa2001316 Medline

72. H. Nishiura, N. M. Linton, A. R. Akhmetzhanov, Serial interval of novel coronavirus (COVID-19) infections. Int. J. Infect. Dis. 93, 284-286 (2020). doi:10.1016/jijij.2020.02.060 Medline

73. Q. Bi, Y. Wu, S. Mei, C. Ye, X. Zou, Z. Zhang, X. Liu, L. Wei, S. A. Truelove, T. Zhang, W. Gao, C. Cheng, X. Tang, X. Wu, Y. Wu, B. Sun, S. Huang, Y. Sun, J. Zhang, T. Ma, J. Lessler, T. Feng, Epidemiology and transmission of COVID-19 in 391 cases and 1286 of their close contacts in Shenzhen, China: A retrospective cohort study. Lancet Infect. Dis. 20, 911-919 (2020). doi:10.1016/S14733099(20)30287-5 Medline

74. J. Mossong, N. Hens, M. Jit, P. Beutels, K. Auranen, R. Mikolajczyk, M. Massari, S. Salmaso, G. S. Tomba, J. Wallinga, J. Heijne, M. Sadkowska-Todys, M. Rosinska, W. J. Edmunds, Social contacts and mixing patterns relevant to the spread of infectious diseases. PLOS Med. 5, e74 (2008). doi:10.1371/journal.pmed.0050074 Medline

75. Office for National Statistics, "Population estimates for the UK, England and Wales, Scotland and Northern Ireland: Mid-2018" (2019); www.ons.gov.uk/peoplepopulationandcommunity/populationandmigration/pop ulationestimates/bulletins/annualmidyearpopulationestimates/mid2018.

\section{ACKNOWLEDGMENTS}

Three anonymous reviewers gave helpful suggestions. We thank Public Health England, COG-UK Consortium volunteers (UK); the Danish Covid-19 Genome Consortium, the Statens Serum Institut (DK); Christian Althaus, Tanja Stadler, Lorenz Risch, the Geneva University Hospitals, the Swiss Viollier Sequencing Consortium at ETH Zürich, the Risch laboratory, the University Hospital Basel, the Institute for Infectious Diseases at University of Bern, the Swiss National Covid-19 Science Task Force (CH); and Helix OpCo, LLC (US) for providing data. Sharon Peacock, Ewan Harrison, Mads Albertsen, Christian Althaus, Tanja Stadler, Lorenz Risch, and Karthik Gangavarapu facilitated data access. Alex Selby suggested improvements to the analysis code. Troy Day gave useful advice for calculating selective benefit and transmission advantage. Stefan Flasche, Rein Houben, Stéphane Hué, Yalda Jafari, Mihály Koltai, Fabienne Krauer, Yang Liu, Rachel Lowe, Billy Quilty, and Julián Villabona Arenas gave input during conception and manuscript drafting. Funding: NGD: UK Research and Innovation (UKRI) Research England; National Institute for Health Research (NIHR) Health Protection Research Unit in Immunisation (NIHR200929); UK Medical Research Council (MRC) (MC_PC_19065). SA: Wellcome Trust (WT) (210758/Z/18/Z). RCB: European Commission (EC) (EpiPose 101003688). CIJ: Global Challenges Research Fund managed through Research Councils UK and the Economic and Social Research Council (RECAP ES/P010873/1). AJK: WT (206250/Z/17/Z); NIHR (NIHR200908). JDM: WT (210758/Z/18/Z). CABP: Bill \& Melinda Gates Foundation (BMGF) (OPP1184344); UK Foreign, Commonwealth and Development Office (FCDO) / WT (221303/Z/20/Z). TWR: WT (206250/Z/17/Z). AG: EC (EpiPose 101003688). WW: NIHR (COV0335), MRC (MR/V027956/1). KVZ: 
FCDO / WT (221303/Z/20/Z); Elrha's Research for Health in Humanitarian Crises Programme funded by FCDO, WT, and NIHR. KDO: Royal Society-WT Sir Henry Dale Fellowship 218554/Z/19/Z. RHK: UKRI Future Leaders Fellowship (MR/S017968/1). RME: Health Data Research UK (MR/S003975/1); MRC (MC_PC 19065); NIHR (NIHR200908). SF: WT (210758/Z/18/Z); NIHR (NIHR200908). MJ: BMGF (INV003174, INV-016832); NIHR (16/137/109, NIHR200929, NIHR200908); EC (EpiPose 101003688). KEA: European Research Council (757688). WJE: EC (EpiPose 101003688); NIHR (NIHR200908). COG-UK is supported by funding from the MRC, part of UKRI; the NIHR; and Genome Research Limited, operating as the Wellcome Sanger Institute. Author contributions: NGD, SA, RCB, CIJ, AJK, JDM, CABP, TWR, DCT, ADW, TW, AG, WW, KLMW, KvZ, JDS, KDO, RK, RME, SF, MJ, KEA, and WJE conceived the study, performed analyses, and wrote the manuscript. NGD led the transmission model analysis, $\mathrm{CIJ}$ led the mobility analysis, ADW led the relativized growth rate analysis, TW led the GLMM analysis, and SA and SF led the $R_{t}$ analysis. The CMMID COVID-19 Working Group provided discussion and comments. Competing interests: ADW owns Selva Analytics LLC. All other authors declare no competing interests. Data and materials availability: All analysis code and data have been archived with Zenodo (43). Code and data for the negative binomial state-space model, multinomial and binomial mixed models and transmission dynamic model are maintained at www.github.com/nicholasdavies/newcovid, and code and data for the $R_{t}$ analysis are maintained at https://github.com/epiforecasts/covid19.sgene. utla.rt. This work is licensed under a Creative Commons Attribution 4.0 International (CC BY 4.0) license, which permits unrestricted use, distribution, and reproduction in any medium, provided the original work is properly cited. To view a copy of this license, visit https://creativecommons.org/licenses/by/4.0/. This license does not apply to figures/photos/artwork or other content included in the article that is credited to a third party; obtain authorization from the rights holder before using such material.

\section{SUPPLEMENTARY MATERIALS}

science.sciencemag.org/cgi/content/full/science.eabg3055/DC1

Materials and Methods

Supplementary Text

Figs. S1 to S24

Tables S1 to S6

References (44-75)

MDAR Reproducibility Checklist

23 December 2020; accepted 26 February 2021

Published online 3 March 2021

10.1126/science.abg3055 
Table 1. Estimates of increased reproduction number for VOC 202012/01. Means and 95\% Cls (GLMM) $/ 95 \%$ Crls $\left(R_{t}\right.$ regression, transmission model) shown. GLMM models do not estimate a baseline growth rate or reproduction number. Increases in the reproduction number assume a generation interval of 5.5 days. See table $\mathrm{S} 1$ for full details.

\begin{tabular}{|c|c|c|c|c|c|c|c|}
\hline Model type & & Model assumptions & Data & Geography & $\begin{array}{l}\text { Baseline growth } \\
\text { rate }\end{array}$ & $\begin{array}{l}\text { Additive increase } \\
\text { in growth rate, } \Delta r\end{array}$ & $\begin{array}{l}\text { Baseline } \\
\text { reproduction } \\
\text { number }\end{array}$ \\
\hline GLMM & $1 \mathrm{la}$ & $\begin{array}{l}\text { Separate-slopes } \\
\text { multinomial spline } \\
\text { model* }\end{array}$ & Sequence & Regions of UK & - & $\begin{array}{c}0.104 \\
{[0.100,0.108]}\end{array}$ & - \\
\hline GLMM & $1 b$ & $\begin{array}{l}\text { Common-slope } \\
\text { multinomial model* }\end{array}$ & Sequence & $\begin{array}{l}\text { Lower-tier local authorities } \\
\text { of UK }\end{array}$ & - & $\begin{array}{c}0.093 \\
{[0.091,0.095]}\end{array}$ & - \\
\hline GLMM & $2 \mathrm{~h}$ & $\begin{array}{l}\text { Separate-slope } \\
\text { binomial spline } \\
\text { modelt }\end{array}$ & $\begin{array}{l}\text { S gene target } \\
\text { failureł }\end{array}$ & Regions of England & - & $\begin{array}{c}0.109 \\
{[0.107,0.111]}\end{array}$ & - \\
\hline$R_{t}$ regression & $4 a$ & $\begin{array}{l}\text { Regional time- } \\
\text { varying baseline }\end{array}$ & $\begin{array}{l}\text { S gene target } \\
\text { failure }\end{array}$ & $\begin{array}{l}\text { Upper-tier local authorities } \\
\text { of England }\end{array}$ & $\begin{array}{c}0.007 \\
{[0.002,0.012]}\end{array}$ & $\begin{array}{c}0.067 \\
{[0.060,0.073]}\end{array}$ & $\begin{array}{c}1.04 \\
{[1.01,1.07]}\end{array}$ \\
\hline$R_{t}$ regression & $4 b$ & $\begin{array}{l}\text { Regional static } \\
\text { baseline }\end{array}$ & $\begin{array}{l}\text { S gene target } \\
\text { failure }\end{array}$ & $\begin{array}{l}\text { Upper-tier local authorities } \\
\text { of England }\end{array}$ & $\begin{array}{c}0.007 \\
{[0.002,0.012]}\end{array}$ & $\begin{array}{c}0.085 \\
{[0.079,0.091]}\end{array}$ & $\begin{array}{c}1.04 \\
{[1.01,1.07]}\end{array}$ \\
\hline $\begin{array}{l}\text { Transmission } \\
\text { model }\end{array}$ & $5 a$ & $\begin{array}{l}\text { Increased } \\
\text { transmissibility }\end{array}$ & $\begin{array}{l}\text { S gene target } \\
\text { failuref }\end{array}$ & Regions of England & $\begin{array}{c}-0.001 \\
{[-0.017,0.012]}\end{array}$ & $\begin{array}{c}0.118 \\
{[0.067,0.168]}\end{array}$ & $\begin{array}{c}1.01 \\
{[0.94,1.09]}\end{array}$ \\
\hline GLMM & $3 a$ & $\begin{array}{l}\text { Common-slope } \\
\text { binomial model } \dagger\end{array}$ & Sequence & Regions of Denmark & - & $\begin{array}{c}0.080 \\
{[0.067,0.092]}\end{array}$ & - \\
\hline GLMM & $3 b$ & $\begin{array}{l}\text { Common-slope } \\
\text { binomial model } \dagger\end{array}$ & $\begin{array}{l}\text { Sequence + } \\
\text { RT-PCR } \\
\text { rescreening }\end{array}$ & Regions of Switzerland & - & $\begin{array}{c}0.101 \\
{[0.092,0.109]}\end{array}$ & - \\
\hline GLMM & $3 c$ & $\begin{array}{l}\text { Common-slope } \\
\text { binomial model } \dagger\end{array}$ & $\begin{array}{l}\text { S gene target } \\
\text { failuref }\end{array}$ & States of USA & - & $\begin{array}{c}0.084 \\
{[0.080,0.088]}\end{array}$ & - \\
\hline
\end{tabular}

*VOC 202012/01 versus B.1.177. †VOC 202012/01 versus all other variants. $\ddagger$ Binomial counts adjusted for the true positive rate (proportion of S gene target failures that are VOC 202012/01), estimated from misclassification model (for UK) or a binomial GLMM fitted to sequencing data of S gene target failures (for US).

Table 2. Summary of projections for England, 15 December 2020-30 June 2021.

\begin{tabular}{|c|c|c|c|c|}
\hline & Moderate (October 2020) & $\begin{array}{l}\text { High (November 2020) with } \\
\text { schools open }\end{array}$ & High with schools closed & Very high (March 2020) \\
\hline \multicolumn{5}{|l|}{ No vaccination } \\
\hline Peak ICU (relative to 1st wave) & $274 \%(256-292 \%)$ & $162 \%(151-172 \%)$ & $130 \%(122-136 \%)$ & $119 \%(112-124 \%)$ \\
\hline Peak ICU bed requirement & $9,980(9,330-10,600)$ & $5,880(5,490-6,280)$ & $4,720(4,450-4,960)$ & $4,310(4,070-4,530)$ \\
\hline Peak deaths & $3,960(3,730-4,200)$ & $2,050(1,920-2,160)$ & $1,500(1,440-1,570)$ & $1,830(1,670-2,000)$ \\
\hline Total admissions & $635,000(604,000-659,000)$ & $454,000(432,000-472,000)$ & $448,000(425,000-466,000)$ & $450,000(425,000-472,000)$ \\
\hline Total deaths & $216,000(205,000-227,000)$ & $146,000(138,000-152,000)$ & $147,000(139,000-155,000)$ & $149,000(140,000-157,000)$ \\
\hline \multicolumn{5}{|l|}{200,000 vaccinations per week } \\
\hline Peak ICU (relative to 1st wave) & $269 \%(252-287 \%)$ & $160 \%(149-170 \%)$ & $130 \%(122-136 \%)$ & $118 \%(112-124 \%)$ \\
\hline Peak ICU bed requirement & $9,790(9,150-10,400)$ & $5,810(5,430-6,200)$ & 4,710 & $0-4,520)$ \\
\hline Peak deaths & $3,700(3,500-3,920)$ & $1,930(1,820-2,040)$ & $1,490(1,430-1,550)$ & $1,320(1,280-1,380)$ \\
\hline Total admissions & $610,000(580,000-634,000)$ & $438,000(416,000-454,000)$ & $415,000(394,000-430,000)$ & $394,000(373,000-413,000)$ \\
\hline Total deaths & $202,000(192,000-213,000)$ & $137,000(130,000-143,000)$ & $129,000(123,000-135,000)$ & $119,000(112,000-125,000)$ \\
\hline \multicolumn{5}{|l|}{2 million vaccinations per week } \\
\hline Peak ICU (relative to 1st wave) & $236 \%(221-252 \%)$ & $149 \%(139-158 \%)$ & $128 \%(121-134 \%)$ & $118 \%(111-124 \%)$ \\
\hline Peak ICU bed requirement & $8,590(8,050-9,170)$ & $5,400(5,070-5,760)$ & $4,650(4,390-4,880)$ & $4,290(4,060-4,500)$ \\
\hline Peak deaths & $2,470(2,330-2,610)$ & $1,510(1,450-1,580)$ & $1,390(1,340-1,450)$ & $1,290(1,250-1,340)$ \\
\hline Total admissions & $483,000(459,000-502,000)$ & $353,000(337,000-366,000)$ & $277,000(265,000-287,000)$ & $190,000(182,000-197,000)$ \\
\hline Total deaths & $140,000(133,000-146,000)$ & $98,900(94,600-103,000)$ & $81,000(77,600-84,200)$ & $58,200(56,100-60,300)$ \\
\hline
\end{tabular}




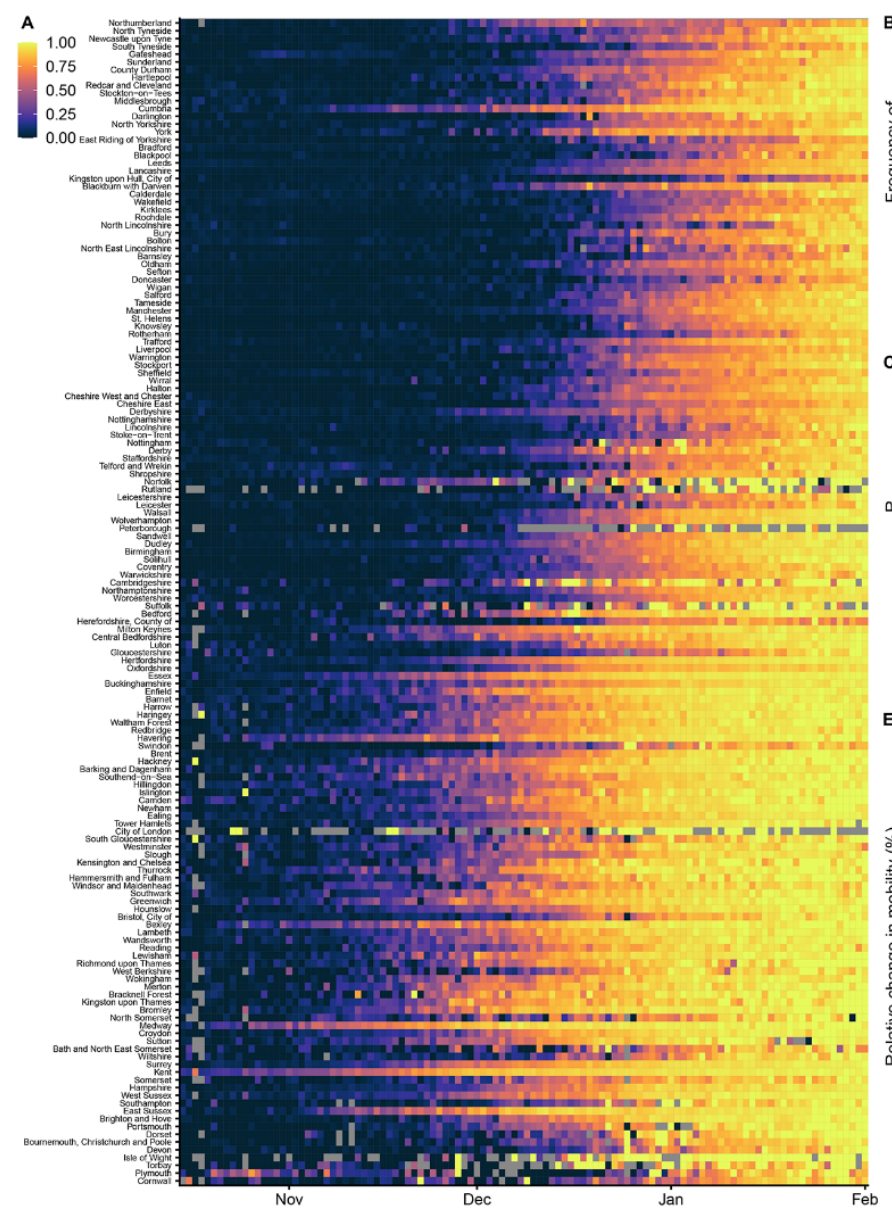

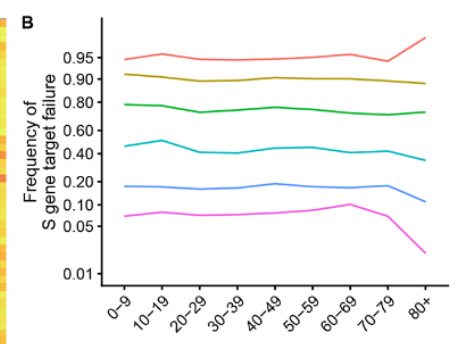
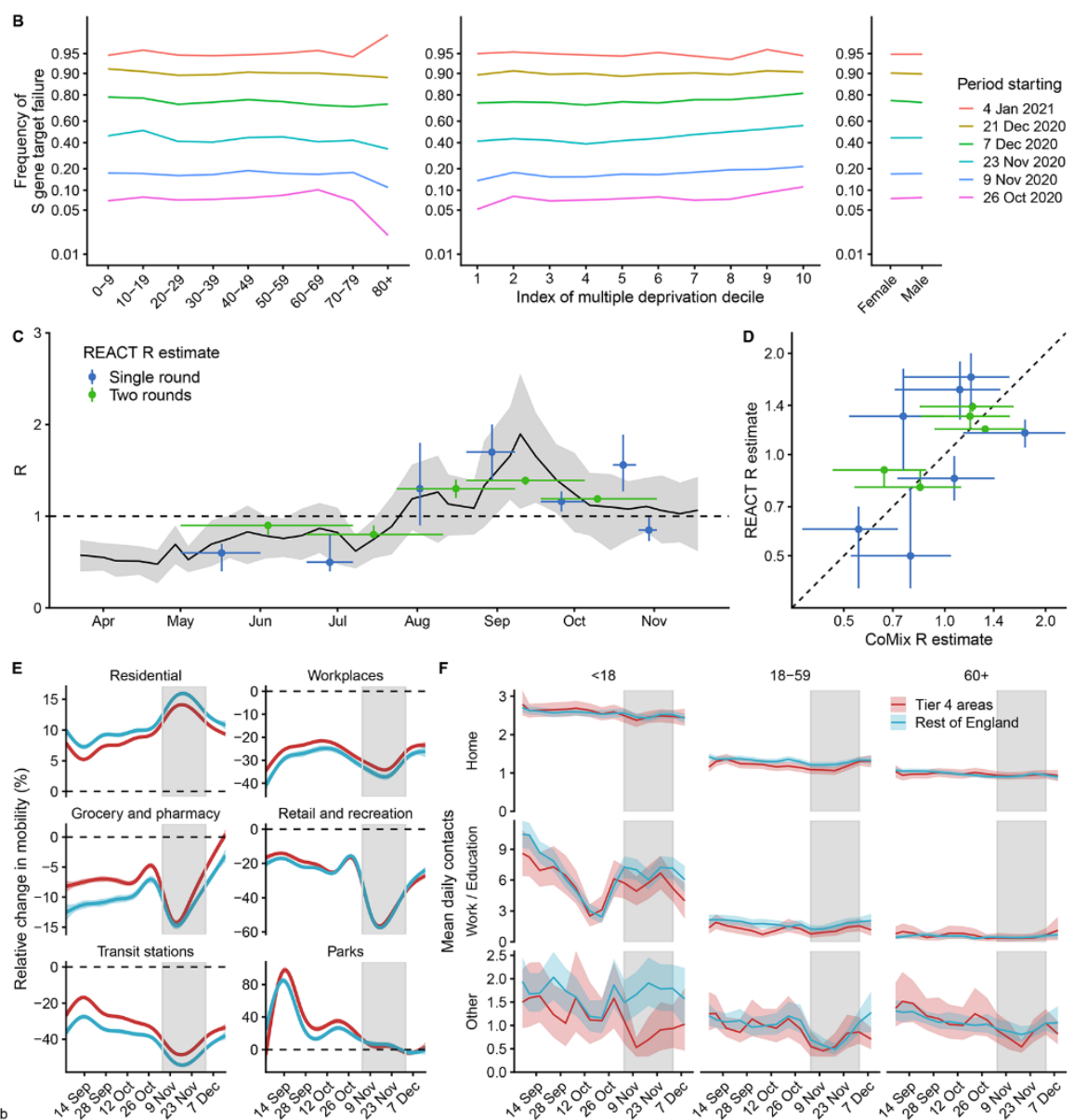

Fig. 1. Rapid spread of VOC 202012/01 in England. (A) Proportion of S gene target failure among positive Pillar 2 community SARS-CoV-2 tests in upper-tier local authorities of England from 1 October 2020-10 January 2021, sorted by latitude. (B) Spread of S gene target failure by age, index of multiple deprivation decile $(1=$ most deprived), and sex within Greater London. (C and D) Estimates of $R_{0}$ from CoMix social contact survey (12) compared to $R_{\mathrm{t}}$ estimates from REACT-1 prevalence survey (9) for England, with $90 \%$ Cls. $R_{t}$ estimates based on single and aggregated REACT-1 survey rounds are shown. (E) Percentage change $(95 \% \mathrm{Cl})$ in Google Mobility indices relative to baseline over time and (F) setting-specific mean contacts $(95 \% \mathrm{Cl})$ from the CoMix study (12) over time and by age for Tier 4 local authorities compared to the rest of England. Tier 4 local authorities are areas within the South East, East of England, and London regions that were placed under stringent restrictions from 20 December 2020 due to high prevalence of VOC 202012/01 and growing case rates. Grey shaded areas show the second national lockdown in England. 

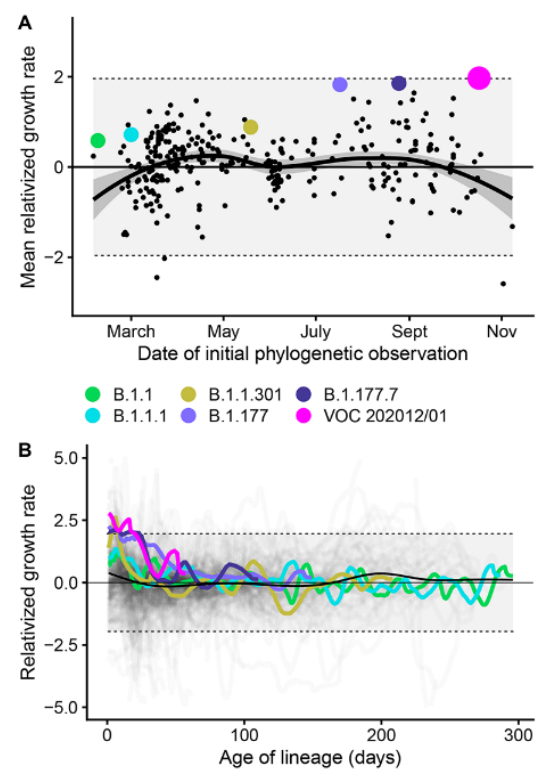

C

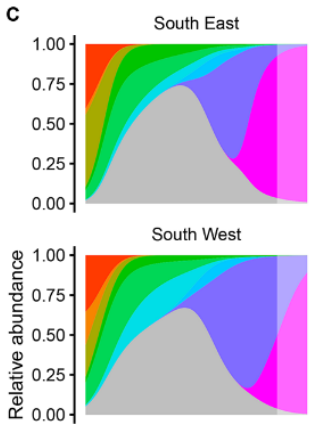

Scotland

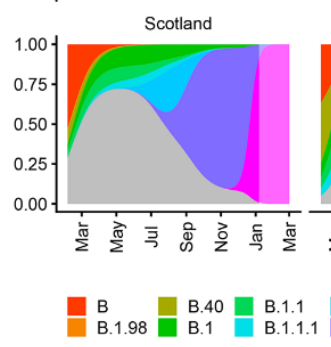

London

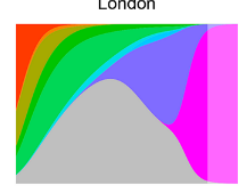

Midlands

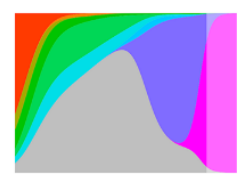

North West

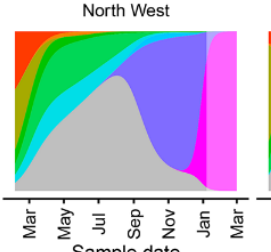

Sample date

B.1.1.315
B.1.177

B.1.1.315
B.1.177

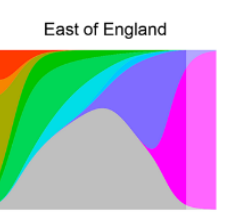

North East and Yorkshire

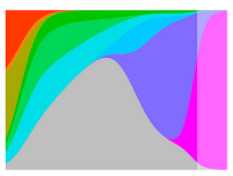

Wales

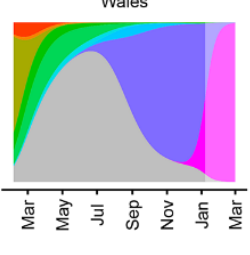

VOC 202012/01
minority variants

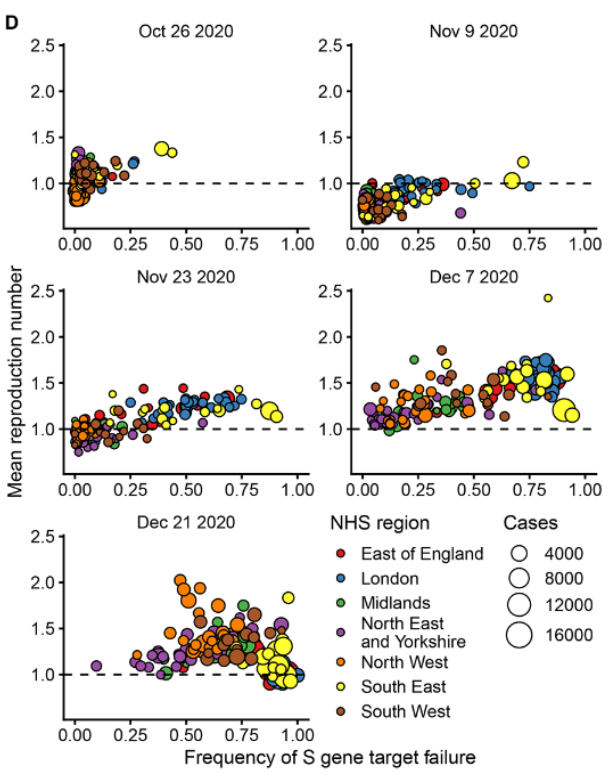

Fig. 2. Measuring the growth rate of VOC 202012/01. (A) Average relativized growth rate, i.e., a measure of variant fitness relative to other variants present during the 31 days following initial phylogenetic observation of a given variant, for all lineages in the COG-UK dataset, highlighting many lineages that have risen to prominence including B.1.177, the lineage with the highest relative abundance during the IPO of VOC 202012/01. The shaded regions show conservative 95\% rejection intervals; VOC 202012/01 is the first strain to exceed this threshold of faster relativized growth. While many lineages exhibit above-average rates of growth, VOC 202012/01 has had the highest average relativized growth of any lineage in the history of COG-UK surveillance of SARS-COV-2. (B) Plotting all lineages' relativized growth rates $[\rho(t)]$ as a function of lineage age with conservative 95\% rejection intervals highlights the significantly faster growth of VOC 202012/01 relative to other lineages at comparable times since their initial observation. Later declines in VOC and B.1.177 correspond to highly uncertain estimates of growth rates for data that are yet to be backfilled, and so these declines in $\rho(t)$ are sensitive to the processing of future sequences from recent dates (fig. S1). (C) Muller plots of the relative abundances of the major SARS-CoV-2 variants in the UK, based on a multinomial spline fit to COG-UK sequence data (Table 1 and table S1, separate-slopes multinomial spline model). A model extrapolation until March 1 is shown (shaded area). Minority variants are 440 circulating SARS-CoV-2 variants of low abundance. Specific colors represent the same lineages in panels A-C. (D) Mean reproduction number over 7-day periods in 149 upper-tier local authorities of England (colored by the NHS England region they are within) plotted against the weekly proportion of Pillar 2 community SARS-CoV-2 tests with S gene target failure shows the spread of VOC 202012/01, a corresponding increase in the reproduction number by local authority, and the eventual impact of targeted government restrictions from 20 December 2020. Testing data are shown for the week following the reproduction number estimates to account for delays from infection to test. 

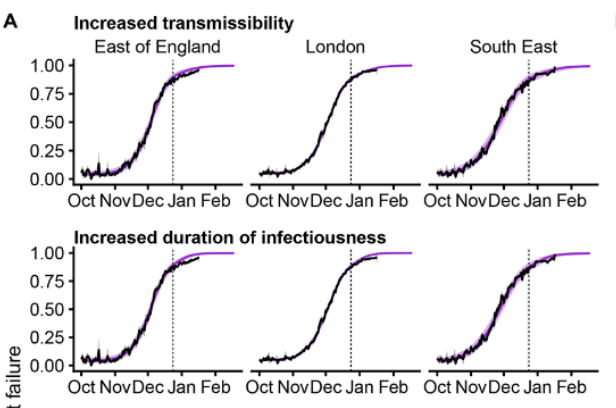

Immune escape

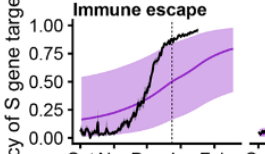

ব্ 0.00 Oct NovDec Jan Feb Oct NovDec Jan Feb Oct NovDec Jan Feb

Increased susceptibility in children

这 1.00

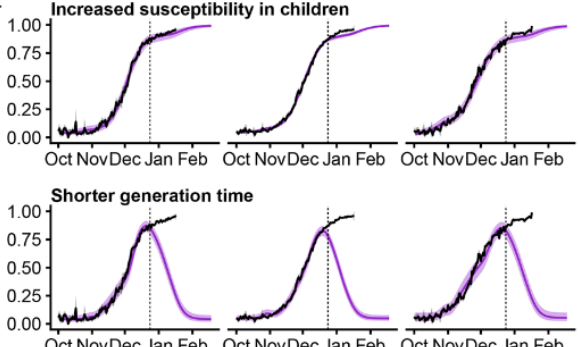

Oct NovDec Jan Féb Oct NovDec Jan Féb Oct NovDec Jan Féb
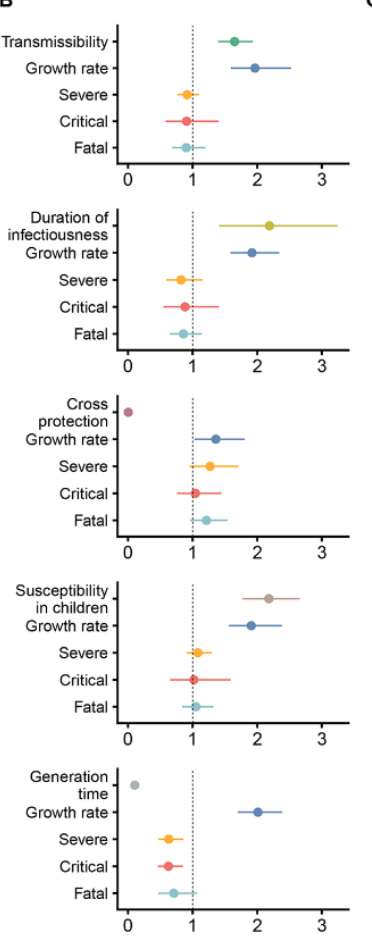

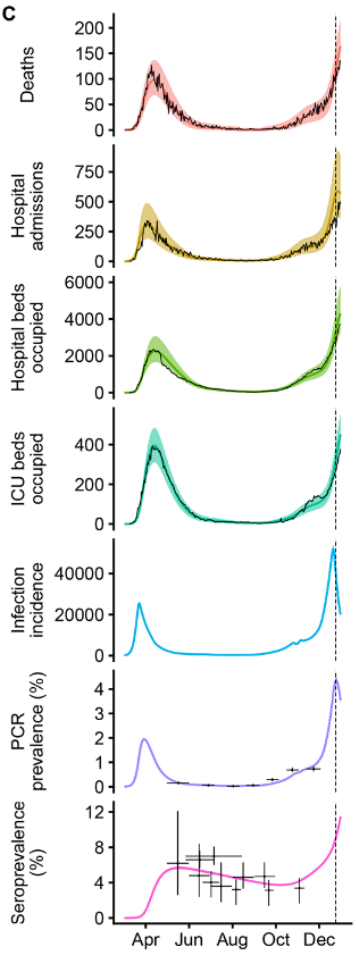

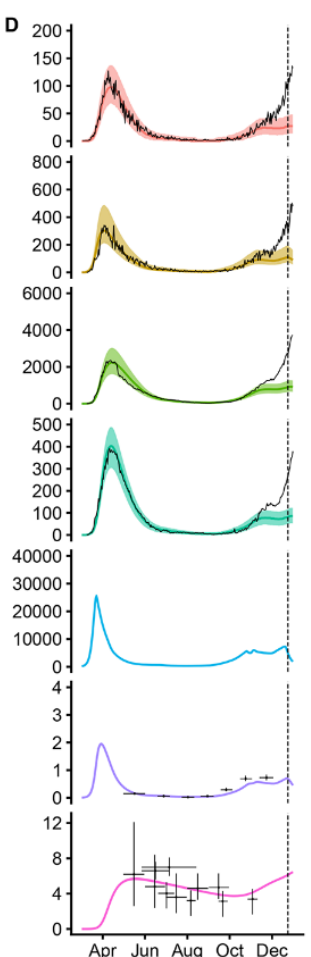

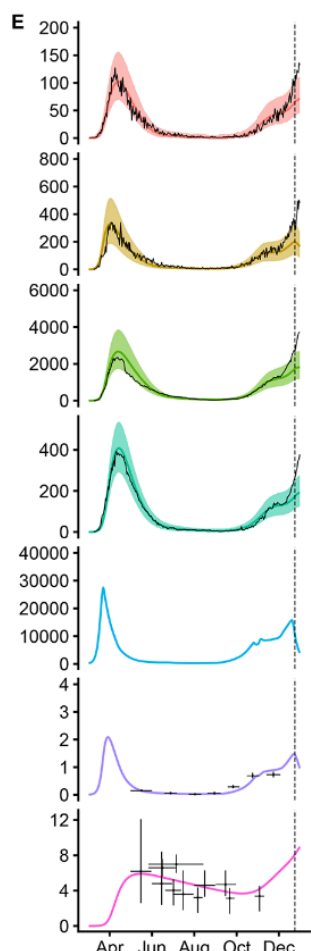

Fig. 3. Comparison of possible biological mechanisms underlying the rapid spread of VOC 202012/01. Each row shows a different assumed mechanism. (A) Relative frequency of VOC 202012/01 (black line and ribbon shows observed S gene target failure frequency with 95\% binomial credible interval; purple line and ribbon shows mean and 95\% credible interval from model fit). (B) Posterior estimates for relative odds of hospitalisation (severe illness), relative odds of ICU admission (critical illness), relative odds of death (fatal illness), growth rate as a multiplicative factor per week (i.e., $\exp (7 \cdot \Delta r)$ ), and the parameter that defines the hypothesized mechanism; all parameters are relative to those estimated for preexisting variants. Illustrative model fits for the South East NHS England region: (C) fitted two-strain increased transmissibility model with VOC 202012/01 included; (D) fitted two-strain increased transmissibility model with VOC 202012/01 removed; (E) fitted singlestrain model without emergence of VOC 202012/01. 
A

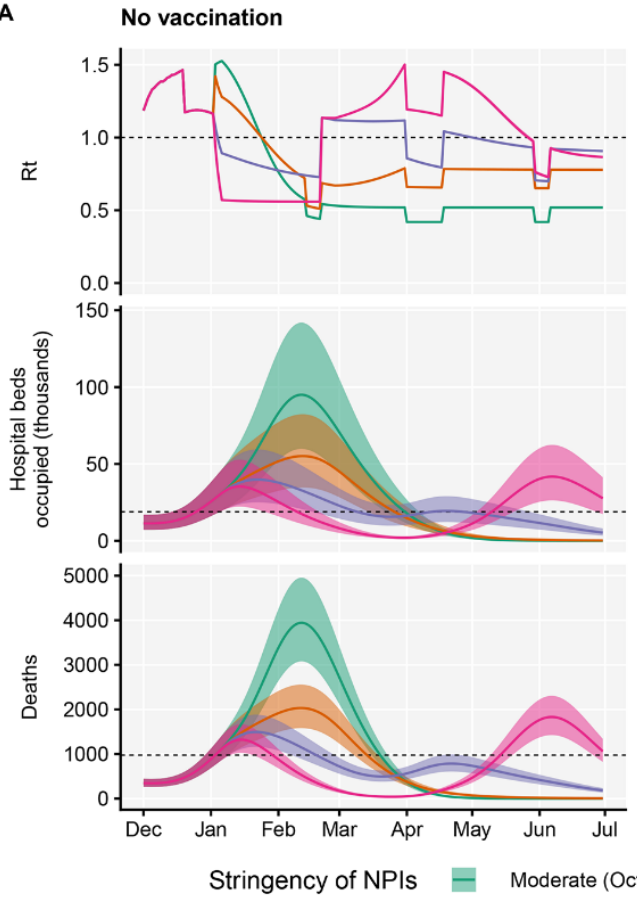

B

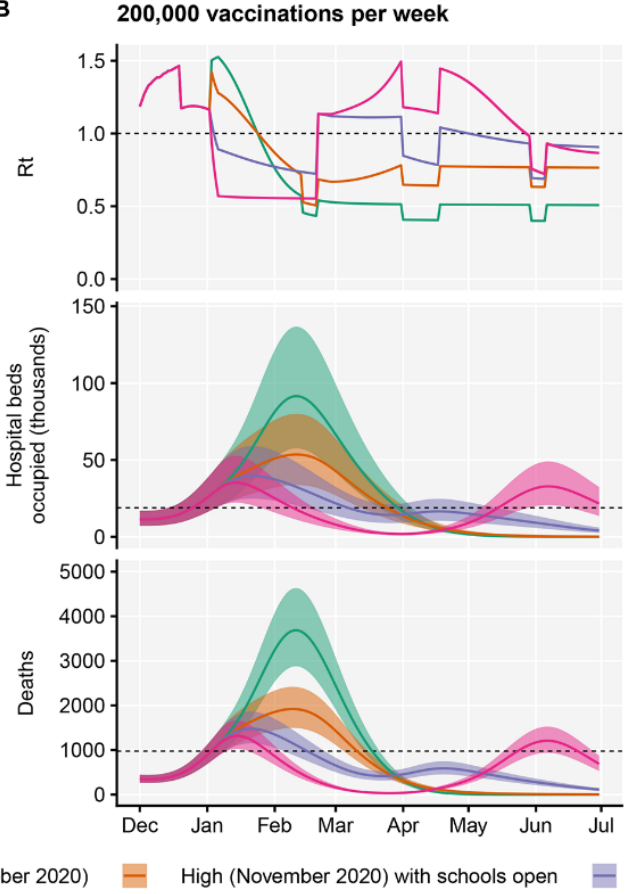

c

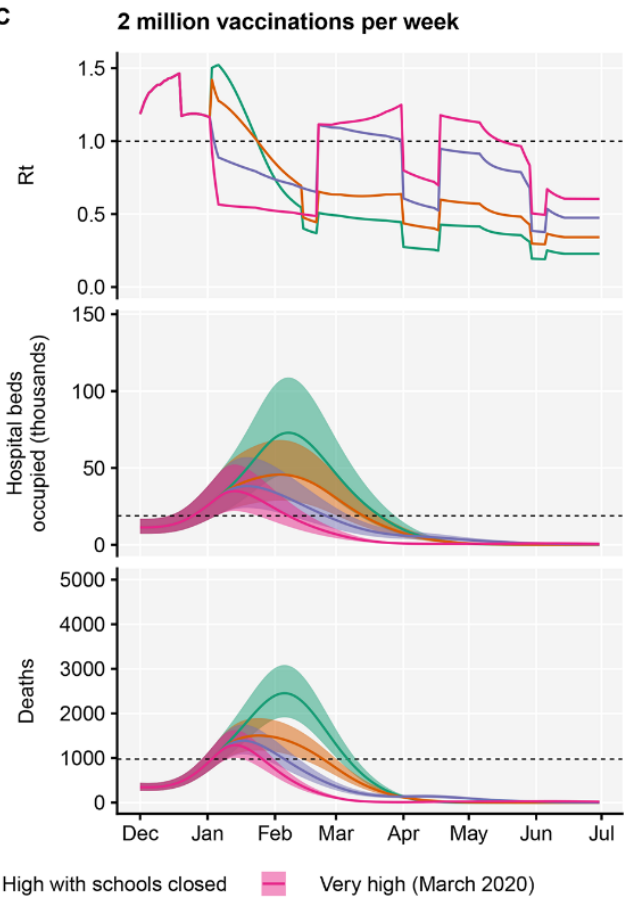

Fig. 4. Projections of epidemic dynamics under different control measures. We compare four alternative scenarios for non-pharmaceutical interventions from 1 January 2021: (i) mobility returning to levels observed during relatively moderate restrictions in early October 2020; (ii) mobility as observed during the second lockdown in England in November 2020, then gradually returning to October 2020 levels from 1 March to 1 April 2021, with schools open; (iii) as (ii), but with school closed until 15 February 2021; (iv) as (iii), but with a lockdown of greater stringency as observed in March 2020 (fig. S20). (A) Without vaccination. (B) With 200,000 people vaccinated per week. (C) With 2 million people vaccinated per week. We assume that vaccination confers $95 \%$ vaccine efficacy against disease and $60 \%$ vaccine efficacy against infection, and that vaccination starts on 1 January 2021 with vaccine protection starting immediately upon receipt. This is intended to approximate the fact that vaccination started in early December, but that full protection occurs after a time lag and potentially after a second dose. Vaccines are given first to $70+$ year olds until $85 \%$ coverage is reached in this age group, then to $60+$ year olds until $85 \%$ coverage is reached in this age group, continuing into younger age groups in 10-year decrements. Resurgences starting in March 2021 are due to the relaxation of non-pharmaceutical interventions, including reopening schools (fig. S20). Median and 95\% credible intervals are shown. The dotted lines in rows 2 and 3 show peak hospitalisations and deaths from the first COVID-19 wave in England (April 2020). 